\title{
GLOBAL DIMENSION OF TILED ORDERS OVER COMMUTATIVE NOETHERIAN DOMAINS
}

\author{
BY
}

\author{
VASANTI A. JATEGAONKAR(1)
}

ABSTRACT. Let $R$ be a commutative noetherian domain and $\Lambda=\left(\Lambda_{i j}\right) \subseteq$ $M_{n}(R)$ be a tiled $R$-order. The main result of this paper is the following

Theorem. Let $\mathrm{gl} \operatorname{dim} R=d<\infty$ and $\mathrm{A}$ a triangular tiled $R$-order (i.e., $\Lambda_{i j}=R$ whenever $\left.i \leq j\right)$. Then the following three conditions are equivalent:

(1) $\mathrm{gl} \operatorname{dim} \mathrm{A}<\infty$;

(2) $\Lambda_{i, i-1}=R$ or $\mathrm{gl} \operatorname{dim}\left(R / \Lambda_{i, i-1}\right)<\infty$, whenever $2 \leq i \leq n$;

(3) $g l \operatorname{dim} \Lambda \leq d(n-1)$.

If $d=1$ or 2 then the upper bound in the above theorem is best possible. We give a sufficient condition for an arbitrary tiled $R$-order $\Lambda$ to be of finite global dimension.

Introduction. Throughout this paper $R$ denotes a commutative noetherian domain with quotient field $K$. As usual, an $R$-order $\Lambda$ in the $n \times n$ matrix ring $M_{n}(K)$ is an $R$-subalgebra of $M_{n}(K)$ which is finitely generated as an $R$-module and spans $M_{n}(K)$ over $K$. The object of this paper is to establish an intimate connection between the finiteness of global dimension of $\Lambda$ and the structure of $\Lambda$, in case $\Lambda$ is a tiled $R$-order contained in $M_{n}(R)$.

An $R$-order $\Lambda$ in $M_{n}(K)$ is tiled if it contains $n$ orthogonal idempotents. Clearly $\Lambda$ is left as well as right noetherian. A certain amount of normalization is possible. Thus, by conjugating if necessary, we may assume that $\Lambda$ contains the idempotents $e_{i i}, 1 \leq i \leq n$, where $\left\{e_{i j}: 1 \leq i, j \leq n\right\}$ is the system of usual matrix units in $M_{n}(K)$. Evidently, the $(i, j)$ th entries of all elements of $\Lambda$ form a nonzero fractional $R$-ideal of $K$, which we shall denote as $\Lambda_{i j^{*}}$ It is equally clear that $R \subseteq \Lambda_{i i}, \Lambda_{i j} \Lambda_{j k} \subseteq \Lambda_{i k}$ and $\Lambda=\left(\Lambda_{i j}\right)$. Note that if $\Lambda=\left(\Lambda_{i j}\right) \subseteq M_{n}(R)$, then all $\Lambda_{i j}$ are integral and $\Lambda_{i i}=R$.

The present author ([8], [9]) and independently R. B. Tarsey [18] have shown

Received by the editors May 17, 1973.

AMS (MOS) subject classifications (1970). Primary 16A60, $13 \mathrm{H} 05$.

Key words and phrases. Orders, tiled orders over commutative noetherian domains, global dimension, regular local rings.

(1) This paper contains a part of the author's Ph.D thesis written at Cornell University under the direction of Professor Alex Rosenberg, whom the author wishes to thank for his help and encouragement.

Copyrieht O 1974, American Mathematical Society 
that if $R$ is a discrete valuation ring (DVR) with maximal ideal $m$ and if $\Lambda=$ $\left(\Lambda_{i j}\right) \subseteq M_{n}(R)$ is a triangular tiled $R$-order (i.e., $\Lambda_{i j}=R$ whenever $i \leq j$ ), then gl $\operatorname{dim} \Lambda<\infty$ if and only if $\mathfrak{m} \subseteq \Lambda_{i, i-1}$ for all $i$. The present author ([8], [9]) gave a sharp upper bound, viz., $n-1$, on their global dimension. The main result (Theorem 3.6) shows that these results can be retained in a suitably modified form even when $R$ is an arbitrary noetherian domain of finite global dimension. The sufficient condition for finiteness of $\mathrm{gl} \operatorname{dim} \Lambda$, obtained in $\$ 2$, is of independent interest (Theorem 2.7).

Several results from this paper will be needed in the sequel in which we investigate global dimension of arbitrary tiled orders over a DVR.

We now state some known results which will be needed in the following sections.

Lemma 0.1. If $S$ is a ring and $S$ is not semisimple, then

$$
\text { r } g l \operatorname{dim} S=1+\sup _{I}\left\{\text { hd }_{S} I\right\} \text {, }
$$

where the supremum is taken over all right ideals of $S$.

Proof. See [14, p. 178, Theorem 9.14].

Lemma 0.2. Let $S$ be any commutative ring, $\Lambda$ a right noetherian $S$-algebra. Then

$$
\text { r } g l \operatorname{dim} \Lambda=\sup _{n_{\mathfrak{l}}} \mathrm{r} g l \operatorname{dim}\left(\Lambda \underset{S}{\otimes} S_{\mathrm{m}}\right)
$$

where $m$ runs through all maximal ideals of $S$.

Proof. See [1, Corollary to Proposition 2.6].

Lemma 0.3. Let $S$ be any ring, $I$ a two-sided ideal which is projective as a left S-module, and $I^{n}=I^{n+1}$ for some integer $n \geq 1$. If $1 \mathrm{gl} \operatorname{dim} S<\infty$, then $1 \mathrm{gl} \operatorname{dim}(S / I)<\infty$.

Proof. See [4, Theorem 1].

Lemma 0.4: If $S$ is a right noetherian ring and $I$ is any two-sided ideal contained in the Jacobson radical $J(S)$ of $S$, then $\mathrm{r} \mathrm{gl} \operatorname{dim} S \leq \operatorname{lwd}_{S}(S / I)+$ $\mathrm{r} \mathrm{gl} \operatorname{dim}(S / I)$, where $1 \mathrm{wd}$ denotes left weak dimension.

Proof. [16, Theorem 1].

Lemma 0.5. Let $S$ be any ring and $e$ an idempotent in $S$. If $\operatorname{lwd}_{e} e_{e} e S=0$, then for every right eSe-module $N$, we have hd ${ }_{s}\left(N \otimes_{e} S e\right.$ eS) $=h_{e} S e^{N \text {; further- }}$ more, r $\mathrm{gl} \operatorname{dim} e S e \leq \mathrm{r} g l \operatorname{dim} S$.

Proof. See [5, Proposition 15, Theorem 7]. 
Lemma 0.6. Let $S$ be any ring. Let

$$
0-B \rightarrow A-C \rightarrow 0
$$

be a short exact sequence of right $S$-modules. Then:

(1) If two of the dimensions hd $A$, hd $s, h_{s} C$, are finite, then so is the third.

(2) If hd ${ }_{s} A \geqslant$ hd $_{s} B$, then hd $C=$ hd $_{s} A$.

(3) If hd ${ }_{s} A \lesseqgtr$ hd $_{s} B$, then hd $C=1+$ hd $_{s} B$.

(4) If hd ${ }_{s} A=\operatorname{hd}_{s} B$, then hd ${ }_{s} C \leq 1+$ hd $_{s} B$.

Proof. See [11, p. 169, Theorem 2].

Lemma 0.7. Let $S$ be a ring and $x$ a central nonzero divisor in $S$. Write $S^{*}=S /(x)$. Let $A$ be a nonzero $S^{*} \cdot$ module with hd ${ }_{s^{*}} A<\infty$. Then hd $A=$ hd ${ }_{S^{*}} A+1$.

Proof. See [11, p. 172, Theorem 3].

Lemma 0.8. Let $S$ be a right noetherian ring and $x$ a central element in the Jacobson radical $J(S)$ of $S$. Let $A$ be a finitely generated right $S$-module. If $x$ is a nonzero divisor on both $S$ and $A$, then hd $S_{*}(A / A x)=\operatorname{hd}_{S} A$, where $S^{*}=S /(x)$.

Proof. See [11, p. 178, Theorem 9].

Lemma 0.9. Let $R$ be a regular local ring with maximal ideal $m$. Let $I$ be a nonzero proper ideal of $R$. Then, $g 1 \operatorname{dim}(R / I)<\infty$ if and only if $I=\left(x_{1}, x_{2}\right.$, $\left.\cdots, x_{s}\right)$, where the $x_{i}$ form a part of an R-sequence generating $\mathrm{m}$.

Proof. See [11, p. 184, Theorem 13], [19, p. 303, Theorem 26].

Lemma 0.10. Let $R$ be a regular local ring of dimension $d$. Let $I$ be a proper nonzero ideal of $R$. If $\mathrm{gl} \operatorname{dim}(R / I)<\infty$, then $\mathrm{gl} \operatorname{dim}(R / I) \leq d-1$.

Proof. Follows easily from Lemma 0.9.

1. Preliminaries. In this paper all rings are associative and have unit element, all modules are unitary. $J(S)$ will denote the Jacobson radical of the ring $S$. By a principal right (left) projective of $S$ we will mean a right (left) ideal of $S$ generated by an indecomposable idempotent of $S$. If $m$ is a maximal ideal of $S$, then $S_{m}$ will denote the localization of $S$ at $m$. If $M$ is an $S$-module, then hd ${ }_{S} M$ will denote the projective dimension of $M$. If $S$ is noetherian, then the right and the left global dimensions of $S$ are equal and this common value will be denoted by $\mathrm{gl} \operatorname{dim} S$. Mad-S ( $S$-Mad) will denote the category of right (left) $S$-modules. Throughout this paper $R$ will denote a commutative noetherian domain. 
Lemma 1.1. Let $S$ be any ring. Let $M_{i}, u_{1} \leq i \leq u_{2}$, and $N_{i}, u_{1}+1 \leq i \leq u_{2}$, be two families of right S-modules. Assume that

(1) $N_{i}$ is projective for all $i$,

(2) $N_{i+1}+M_{i}=M_{i+1}$ for $u_{1} \leq i \leq u_{2}-1$,

(3) $N_{i+1} \cap M_{i} \simeq M_{i}$ for $u_{1} \leq i \leq u_{2}-1$,

Tben hd $M_{i} M_{i}$ hd $_{s} M_{\alpha_{1}}+i-u_{1}$ for $u_{1} \leq i \leq u_{2}$.

Proof. Follows easily from Lemma 0.6 and by induction.

Lemma 1.2. Let $R$ be a commutative, noetberian local domain and let $\Lambda$ be a tiled $R$-order in $M_{n}(K)$, where $K$ is the quotient field of $R$. Then $\mathrm{gl} \operatorname{dim} \Lambda=$ $1+\operatorname{hd}_{\Lambda} J(\Lambda)$.

\section{Proof. See Corollary 4.6 of [15].}

The next lemma is a very handy tool in computation of the Jacobson radical $J(S)$ of the ring $S$ containing a finite set of orthogonal idempotents with sum 1 [7].

Lemma 1.3. Let $S$ be any ring and let $\left\{e_{i}: 1 \leq i \leq n\right\}$ be a finite set of orthogonal idempotents in $S$ witb sum 1. Let $H_{i j}=\left\{e_{i} s e_{j}: e_{i} \text { se }_{j} S e_{i} \subseteq J\left(e_{i} S e_{i}\right)\right\}_{0}$ Then $H_{i i}=J\left(e_{i} S e_{i}\right)=J(S) \cap e_{i} S e_{i}=e_{i} J(S) e_{i}$ and $J(S)=\bigoplus_{i, j} H_{i j}$.

Proof. First we show that $\Sigma_{i, j} H_{i j} \subseteq J(S)$. Let $e_{i} s e_{j}$ be in $H_{i j}$. By Proposition 3 of $\left[12\right.$, p. 57] it is enough to show that, for all $r$ in $S, 1-e_{i} s e_{j} r$ is right invertible in $S$. Since $e_{i} s e_{j} S e_{i} \subseteq J\left(e_{i} S e_{i}\right)$, therefore by the same Proposition we have $e_{i} z e_{i}$ in $e_{i} S e_{i}$ such that

$$
\left(e_{i}-e_{i} s e_{j} r e_{i}\right)\left(e_{i}-e_{i} z e_{i}\right)=e_{i}
$$

Hence $e_{i} s e_{j} g e_{i} z e_{i}=e_{i} s e_{j} g e_{i}+e_{i} z e_{i}$. Consequently, $\left(1-e_{i} s e_{j} r e_{i}\right)\left(1-e_{i} z e_{i}\right)=1$. Now,

$$
\begin{aligned}
\left(1-e_{i} s e_{j} r\right)\left(1-e_{i} z e_{i}\right) & =1-e_{i} z e_{i}-e_{i} s e_{j} r\left(\sum_{k=1}^{n} e_{k}\right)\left(1-e_{i} z e_{i}\right) \\
& =\left(1-e_{i} z e_{i}\right)-e_{i} s e_{j} r e_{i}\left(1-e_{i} z e_{i}\right)-\sum_{k \neq i} e_{i} s e_{j} r e_{k}\left(1-e_{i} z e_{i}\right) \\
& =1-\sum_{k \neq i} e_{i} s e_{j} r e_{k} .
\end{aligned}
$$

However, since the $e_{i}$ 's are orthogonal, it is immediate that

$$
\left(1-\sum_{k \neq i} e_{i} s e_{j} r e_{k}\right)\left(1+\sum_{k \neq i} e_{i} s e_{j} r e_{k}\right)=1 \text {. }
$$

Hence $1-e_{i} s e, r$ is right invertible in $S$. Thus $\Sigma_{i, j} H_{i j} \subseteq J(S)$. Next we prove the assertion about $H_{i i}$. It is easy to see that $J\left(e_{i} S e_{i}\right)=H_{i i} \subseteq J(S) \cap e_{i} S e_{i}=$ $e_{i} J(S) e_{i}$. Let $e_{i} s e_{i}$ be in $e_{i} J(S) e_{i}$ which is contained in $J(S)$. Hence there is an 
$r$ in $S$ such that $\left(1-e_{i} s e_{i}\right)(1-r)=1$; multiplying on both sides by $e_{i}$ we get $\left(e_{i}-e_{i} s e_{i}\right)\left(e_{i}-e_{i} r e_{i}\right)=e_{i}$. Therefore $e_{i} J(S) e_{i} \subseteq J\left(e_{i} S e_{i}\right)$, by Theorem 1 of $[6$, p. 9].

We now finish the proof of the lemma by showing that $J(S) \subseteq \Sigma_{i, j} H_{i j}$. Let $s \in J(S)$. Then $e_{i} s e_{j} \in J(S)$ for all $i, j$. Hence $e_{i} s e_{j} S e_{i} \subseteq J(S) \cap e_{i} S e_{i}=$ $J\left(e_{i} S e_{i}\right)$. Thus $e_{i} s e_{j}$ is in $H_{i j}$ for all $i, j$. Since $\Sigma_{k=1}^{n} e_{k}=1$, therefore $s=\Sigma_{i, j} e_{i} s e_{j} \in \Sigma_{i, j} H_{i j}$. That the sum is direct is obvious.

Corollary 1.4. Let $e$ be an idempotent in a ring $S$. Then $J(e S e)=e J(S) e=$ $J(S) \cap e S e$.

Definition 1.5. Let $\Lambda=\left(\Lambda_{i j}\right) \subseteq M_{n}(R)$ be a tiled $R$-order. Then $\Lambda$ is called reduced if $\Lambda_{i j} \Lambda_{j i} \subsetneq R$ whenever $i \neq j$; equivalently, $\Lambda_{i j} \subsetneq R$ or $\Lambda_{j i} \subsetneq R$ whenever $i \neq j$. Then

Lemma 1.6. Let $\Lambda=\left(\Lambda_{i j}\right) \subseteq M_{n}(R), \Gamma=\left(\Gamma_{i j}\right) \subseteq M_{n}(R)$ be two tiled R-orders.

(1) $\Lambda$ is reduced if and only if $e_{i i} \Lambda \simeq e_{j j} \Lambda$ as right $\Lambda$-modules, whenever $i \neq j$.

(2) If $R$ is a local domain, $\Lambda$ is reduced, and if $\Lambda$ and $\Gamma$ are isomorphic as rings, then $\Gamma$ is also reduced.

Proof. (1) If $\Lambda$ is not reduced then $\Lambda_{i j}=R=\Lambda_{j i}$ for some $i \neq j$. Since $\Lambda_{i l} \supseteq \Lambda_{i j} \Lambda_{j l}=\Lambda_{j l} \supseteq \Lambda_{j i} \Lambda_{i l}=\Lambda_{i l}$, therefore $\Lambda_{i l}=\Lambda_{j l}$ for all $1 \leq l \leq n$. Hence $e_{i i} \Lambda \simeq e_{j j} \Lambda$.

Conversely, assume that $e_{i i} \Lambda \cong e_{j j} \Lambda$ as right $\Lambda$-modules for some $i \neq j$. Let $\theta: e_{i i} \Lambda \rightarrow e_{j j} \Lambda$ be an isomorphism. Since $e_{i i}^{2}=e_{i i}, \theta\left(e_{i i}\right)=\theta\left(e_{i i}^{2}\right)=\theta\left(e_{i i}\right) e_{i i}$ $\epsilon e_{j i} \Lambda e_{i i}$, so that $\theta\left(e_{i i}\right)=a e_{j i}$ for some unit $a$ in $R$. Hence we have $e_{j j} \Lambda=$

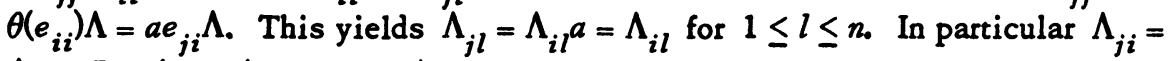
$\Lambda_{i i}=R=\Lambda_{j j}=\Lambda_{i j}$. Thus $\Lambda$ is not reduced.

(2) Since $R$ is local, the $e_{i i}$ 's are local idempotents. Hence, if $\theta: \Lambda \rightarrow \Gamma$ is a ring isomorphism, then by Proposition 3 of $[12, \mathrm{p} .77]$ we may assume that $\theta\left(e_{i i}\right)=e_{\pi(i), \pi(i)}$, where $\pi$ is a permutation of the numbers from 1 to $n$. If $\Gamma$ is not reduced, then, by (1), we have $e_{i i} \Gamma \simeq e_{j j} \Gamma$ as right $\Gamma$-modules for some $i \neq j$. From this, one at once gets that $e_{l l} \Lambda \simeq e_{k k} \Lambda$ as right $\Lambda$-modules, where $l=\pi^{-1}(i)$ and $k=\pi^{-1}(j)$. Thus $\Lambda$ is not reduced.

Lemma 1.7. Let $\Lambda=\left(\Lambda_{i j}\right) \subseteq M_{n}(R)$ be a reduced tiled $R$-order. Then there exist natural numbers $k, l \leq n$ sucb that $\Lambda_{i k} \neq R$ for all $i \neq k$ and $\Lambda_{l i} \neq R$ for all $i \neq l$.

Proof. We shall prove the existence of $k$. The proof for the existence of $l$ is similar. We use induction on $n$. For $n=2$, the assertion is trivial, since 
$\Lambda_{12} \varsubsetneqq R$ or $\Lambda_{21} \varsubsetneqq R$. Let $n \geq 3$. If $\Lambda_{\text {in }} \neq R$ whenever $i \neq n$, then we are done. Suppose that $\Lambda_{j n}=R$ for some $j \leq n-1$. Observe that $e \Lambda e$ is also a reduced tiled $R$-order contained in $M_{n-1}(R)$, where $e=\Sigma_{i=1}^{n-1} e_{i i}$. Hence by the induction hypothesis there is $k \lesseqgtr n$ such that $\Lambda_{i k} \neq R$ whenever $i \neq k, n$. To complete the induction we show that $\Lambda_{n k} \neq R$. If $j=k$, then $\Lambda_{n k}=\Lambda_{n j} \neq R$, since $\Lambda$ is reduced, and we are done. If $j \neq k$, then $\Lambda_{j k} \neq R$ as $j \leq n-1$. If $\Lambda_{n k}=R$, then $\Lambda_{j k}=R$, as $\Lambda_{j k} \supseteq \Lambda_{j n} \Lambda_{n k}=R$. Thus $\Lambda_{n k} \neq R$. This completes the induction and completes the proof of the lemma.

Definition 1.8. Let $\Lambda=\left(\Lambda_{i j}\right) \subseteq M_{n}(R)$ be a tiled $R$-order. $\Lambda$ is called a (super) triangular tiled $R$-order if $\Lambda_{i j}=R$ whenever $i \leq j$.

The next proposition gives a necessary condition for a reduced tiled $R$-order $\Lambda=\left(\Lambda_{i j}\right) \subseteq M_{n}(R), R$ a commutative noetherian local domain with quotient field $K$, to be conjugate to a triangular tiled $R$-order in $M_{n}(K)$.

Let $\Lambda=\left(\Lambda_{i j}\right) \subseteq M_{n}(R)$ be a tiled $R$-order over a commutative noetherian local domain $R$ with maximal ideal $\mathrm{m}$. Let $\bar{\Lambda}=\left(\Lambda_{i j} / \Lambda_{i j} \mathrm{~m}\right)$, where the multiplication in $\bar{\Lambda}$ is induced from that in $\Lambda$, i.e., if $\left(\lambda_{i j}\right),\left(\lambda_{i j}^{\prime}\right) \in\left(\Lambda_{i j}\right)$ then $\left(\lambda_{i j}+\Lambda_{i j} \mathrm{~m}\right)\left(\lambda_{i j}^{\prime}+\Lambda_{i j} \mathrm{~m}\right)=\left(\sum_{k=1}^{n} \lambda_{i k} \lambda_{k j}^{\prime}+\Lambda_{i j} \mathrm{~m}\right)$. Then $\bar{\Lambda}$ is a finite dimensional $R / \mathrm{m}$-algebra which is naturally isomorphic with $\Lambda / \Lambda \mathrm{m}$ as an $R / \mathrm{m}$-algebra. Also, if $\Lambda$ is reduced then, by Lemma 1.3, $I(\bar{\Lambda})$ is obtained from $\bar{\Lambda}$ be replacing the diagonal entries $R / \mathfrak{m}$ by zero. If $M$ is a finitely generated right $\bar{\Lambda}$-module then by Exercise 29 of $[4$, p. 80$]$, we have $J(M)=M J(\bar{\Lambda})$, where $J(M)$ is the intersecttion of all maximal submodules of $M$. Let $\overline{\boldsymbol{e}}_{i i}$ denote the indecomposable idempotent in $\bar{\Lambda}$ with 1 at the $(i, i)$ th place and zero elsewhere.

Proposition 1.9. Let $\Lambda=\left(\Lambda_{i j}\right) \subseteq M_{n}(R)$ be a reduced tiled $R$-order over a commutative noetherian local domain $R$ with maximal ideal $\mathrm{m}$ and quotient field $K$. Let $\bar{P}_{i}=\bar{e}_{i i} \bar{\Lambda}, 1 \leq i \leq n$. Assume that. $\Lambda$ is conjugate to a triangular tiled $R$-order $\Gamma=\left(\Gamma_{i j}\right) \subseteq M_{n}(R)$, i.e., $\Lambda=u \Gamma u^{-1}$ for some unit $u$ in $M_{n}(K)$. Then, for some $j \leq n$,

$$
\bar{P}_{j} \supsetneq \bar{P}_{i} J(\Lambda) \supsetneqq \bar{P}_{j} J^{2}(\Lambda) \supsetneqq \cdots \supsetneqq \bar{P}_{i} J^{n-1}(\Lambda) \supsetneqq \bar{P}_{j} J^{n}(\Lambda)=0
$$

is a composition series of $\bar{P}_{j}$ considered as a right $\bar{\Lambda}$-module.

Proof. Let $\bar{\Gamma}=\left(\Gamma_{i j} / \Gamma_{i j} \mathrm{~m}\right)$, and let $\bar{f}_{i i}$ denote the indecomposable idempotent in $\bar{\Gamma}$ with 1 at the $(i, i)$ th place and zero elsewhere. Let $\bar{Q}_{i}=\bar{f}_{i i} \bar{\Gamma}, 1 \leq$ $i \leq n$. Then $\bar{P}_{i}$ and $\bar{Q}_{i}, 1 \leq i \leq n$, are, up to isomorphism, the only principal right projectives of $\bar{\Lambda}$ and $\bar{\Gamma}$ respectively. Since $\Lambda$ and $\Gamma$ are $R$-isomorphic, $\bar{\Lambda}$ and $\bar{\Gamma}$ are isomorphic as $R / \mathrm{m}$-algebras. Hence to prove the proposition it is enough to show that $\bar{Q}_{1}$ satisfies the condition $(*)$.

By Lemma 1.6(2) we have that $\Gamma$ is reduced, so that $\Gamma_{i j}=R$ for $i \leq j$, 
and $\Gamma_{i j} \subseteq m$ for $i \geqslant j$. Also, by Lemma 1.3 we have $J(\bar{\Gamma})$ is obtained from $\bar{\Gamma}$ by replacing the diagonal entries $R / \mathrm{m}$ by zero. Since the multiplication in $\bar{\Gamma}$ is induced from that in $\Gamma$, it follows that for $i \geqslant j$ we have

$$
\left(\Gamma_{1 i} / \Gamma_{1 i} \mathrm{~m}\right) \cdot\left(\Gamma_{i j} / \Gamma_{i j}^{\mathrm{m}}\right)=0 \quad \text { in }\left(\Gamma_{1 j} / \Gamma_{1 j} \mathrm{~m}\right)=R / \mathrm{m},
$$

and for $i \leq j$ we have

$$
\left(\Gamma_{1 i} / \Gamma_{1 i} \mathfrak{m}\right) \cdot\left(\Gamma_{i j} / \Gamma_{i j} \mathfrak{m}\right)=\left(\Gamma_{1 j} / \Gamma_{1 j} \mathfrak{m}\right)=R / \mathfrak{m}
$$

Now a direct computation shows that

$$
\bar{Q}_{1} \supsetneqq \bar{Q}_{1} J(\Gamma) \supsetneqq \bar{Q}_{1} J^{2}(\bar{\Gamma}) \supsetneqq \cdots \supsetneqq \bar{Q}_{1} J^{n-1}\left(\bar{\Gamma} \supsetneqq \bar{Q}_{1} J^{n}(\bar{\Gamma})=0\right.
$$

is a composition series of $\bar{Q}_{1}$ as a right $\bar{\Gamma}$-module. This completes the proof.

Let $\Lambda=\left(\Lambda_{i j}\right) \subseteq M_{n}(R)$ be a tiled $R$-order. Let $e=\Sigma_{i=1}^{n-1} e_{i i}$. Then $e \Lambda e$ is a tiled $R$-order in $M_{n-1}(R)$. We shall treat $e \Lambda e$ as the $(n-1) \times(n-1)$ tiled $R$-order in $M_{n-1}(R)$ or the top $(n-1) \times(n-1)$ corner interchangeably. Let $P_{i}=e_{i i} \Lambda$ and $J_{i}=e_{i i} J(\Lambda)$ for $1 \leq i \leq n$. Let $\mathcal{F}: M_{a d}-\Lambda \rightarrow M_{a d-e} \Lambda e$ and $\mathcal{G}$ : $\mathbb{M}_{a d-e \Lambda e} \rightarrow M_{a d-\Lambda}$ be the functors defined by $\mathcal{F}_{M}=$ Me and $\mathcal{G}_{N}=N \otimes_{e \Lambda e} e \Lambda$. Then by Lemma 1.3 and Corollary 1.4 we have $J(e \Lambda e)$ is canonically isomorphic to $\bigoplus_{i=1}^{n-1} \mathfrak{F}_{i}$. Since $\Lambda e$ is a projective left $\Lambda$-module and since $M \otimes_{\Lambda} \Lambda e \simeq M e$ under the isomorphism $m \otimes e \mapsto m e$, the functor $\mathcal{F}$ is naturally equivalent to the functor $-\otimes_{\Lambda} \Lambda e$, and therefore is exact and additive. Also, since $e \Lambda \otimes_{\Lambda} \Lambda e \simeq$ $e \Lambda e[2, \mathrm{p} .68]$, the functors $\mathcal{F} \mathcal{G}$ and $1_{\text {Mod-e }}$ e are naturally equivalent. Furthermore, if $e \Lambda e_{n n}$ is a projective left $e \Lambda e$-module, then $e \Lambda=e \Lambda e \oplus e \Lambda e_{n n}$ is a progenerator in $e \Lambda_{e}-M_{0}$, and therefore the functor $\varrho$ is exact and additive; also by Lemma 0.5 we have, for every right $e \Lambda e$-module $N, h_{A} \varrho_{N}=h_{e A e} N$ and $\mathrm{gl} \operatorname{dim} e \Lambda e \leq \mathrm{gl} \operatorname{dim} \Lambda$. Thus we have the following proposition.

Proposition 1.10. Let $R$ be a commutative noetherian domain. Let $\Lambda=$ $\left(\Lambda_{i j}\right) \subseteq M_{n}(R)$ be a tiled $R$-order. Let $e=\Sigma_{i=j}^{n-1} e_{i i}$. If $e \Lambda e_{n n}$ is a projective left $e \Lambda e$-module, then

(1) $\mathcal{F}$ and $\mathcal{G}$ are exact, additive functors; the functors $\mathcal{F} @$ and $1_{\text {mod }}$ Ae are naturally equivalent; $e \Lambda$ is a progenerator in $e \Lambda e-M_{0}$ od $J(e \Lambda e)$ is canonically isomorphic with $\bigoplus_{i=1}^{n-1} \mathfrak{F}_{J_{i}}$.

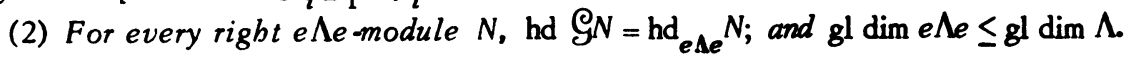

We conclude this section with a few remarks.

Remark 1. If $R$ is a local domain then $\Lambda=\left(\Lambda_{i j}\right)$ is a semiperfect ring, by Theorem 1 of [13]. Hence by using Proposition 3 of [12, p. 77] one can easily show that $e_{i i} \Lambda\left(\Lambda e_{i i}\right), 1 \leq i \leq n$, are, up to isomorphism, the only principal right (left) projectives of $\Lambda$. Thus, by Theorem 2 of [13] or otherwise, every finitely generated indecomposable right (left) $\Lambda$-module is isomorphic to $e_{i i} \Lambda\left(\Lambda e_{i i}\right)$ for some $i$. 
Remark 2. If $R$ is a local domain and if $\Lambda=\left(\Lambda_{i j}\right)$ is reduced, then, by Lemma 1.3, $J(\Lambda)$ is obtained from $\Lambda$ by replacing the diagonal entries $R$ by $m$, the unique maximal ideal of $R$.

Remark 3. If $A=\Sigma_{i=1}^{n} R u_{i}$ is a free left $R$-module on the basis $u_{1}, u_{2}, \cdots, u_{n}$ then $A$ is a right $M_{n}(R)$-module naturally. Since $\Lambda=\left(\Lambda_{i j}\right) \subseteq M_{n}(R)$ is a subring of $M_{n}(R)$, this $M_{n}(R)$-module structure induces an $(R-\Lambda)$-bimodule structure on $A$. Further, if $B$ is a nonzero $\Lambda$-submodule of $A$, then $B=\sum_{i=1}^{n} B_{i} u_{i}$, where $B_{i}$ 's are nonzero ideals of $R$. Hence $A$ is uniform as a right $\Lambda$-module, i.e., if $B$ and $C$ are nonzero $\Lambda$-submodules of $A$, then $B \cap C \neq 0$. Further, $B$ and $x B$ are isomorphic as $\Lambda$-modules whenever $0 \neq x \in R$. Also, a nonzero $\Lambda$-module $B=\sum_{i=1}^{n} B_{i} u_{i}$ of $A$ is isomorphic to $e_{k k} \Lambda$ for some $k \leq n$ if and only if for some $0 \neq x$ in $R$ we have $B_{i}=x \Lambda_{k i}$ for $1 \leq i \leq n$. Similar results are true for a free right $R$-module of rank $n$.

Remark 4. Let $A$ be as in Remark 3. Then $P_{i}=e_{i i} \Lambda, 1 \leq i \leq n$, can be identified with a $\Lambda$-submodule of $A$. This identification makes expressions like $P_{i}+P_{j}, P_{i} \cap P_{j}$ unambiguous. In later sections without mentioning this identification we will use expressions like $P_{i}+P_{j}, P_{i} \cap P_{j}$, etc.

2. A sufficient condition. From now on $R$ will always denote a commutative noetherian domain with the quotient field $K$. Furthermore, if $R$ is local then $m$ will always denote the unique maximal ideal of $R$. We will always assume that a tiled $R$-order $\Lambda=\left(\Lambda_{i j}\right)$ in $M_{n}(K)$ is contained in $M_{n}(R)$. We reserve $e$ for the idempotent $\Sigma_{i=1}^{n-1} e_{i i}$, and $P_{i}=e_{i i} \Lambda, J_{i}=e_{i i} J(\Lambda), 1 \leq i \leq n$.

Lemma 2.1. Let $\Lambda=\left(\Lambda_{i j}\right) \subseteq M_{n}(R)$ be a tiled $R$-order, where $R$ is a local domain. Then,

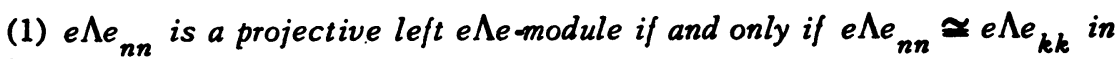

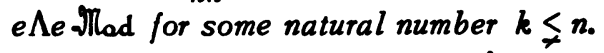

(2) $e \Lambda e_{n n} \simeq e \Lambda e_{k k}$ in $e \Lambda e-M a d$ for some $k \lesseqgtr n$ if and only if there exists $0 \neq a$ in $R$ sucb that $\Lambda_{i n}=\Lambda_{i k} a$ for $1 \leq i \leq n-1$.

Proof. Follows easily from Remarks 1 and 3 at the end of $\$ 1$.

Lemma 2.2. Let $\Lambda=\left(\Lambda_{i j}\right) \subseteq M_{n}(R)$ be a reduced tiled $R$-order over a local

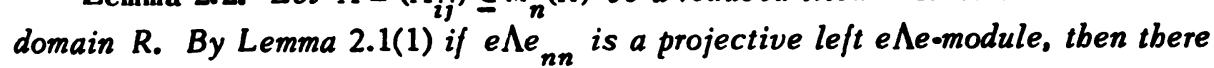

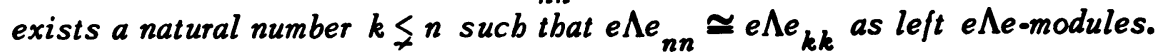
Further,

(1) hd $J_{i}=$ hd $e \Delta e \mathcal{F}_{i}$ for $1 \leq i \leq n-1, i \neq k$.

(2) $\operatorname{hd}_{\Lambda}\left(\mathcal{F}_{j}\right) e \Lambda=\mathrm{hd}_{e \Lambda e} \mathcal{F}_{J_{i}}$ for $i=n, k$.

(3) $\mathcal{F}_{J_{n}}$ is isomorpbic to a right ideal of $e \Lambda e$. 
Proof. By Lemma 2.1(2) we have $0 \neq a$ in $R$ such that $\Lambda_{i n}=\Lambda_{i k} a$ for $1 \leq i \leq n-1$. Therefore $\Lambda_{k n}=R a$ and $a \Lambda_{n i}=\Lambda_{k n} \Lambda_{n i} \subseteq \Lambda_{k i}$ for $1 \leq i \leq n$. By Remark 2 at the end of $\S 1, J(\Lambda)$ is obtained from $\Lambda$ by replacing the diagonal entries $R$ by $m$. It follows that $\theta: \mathcal{F}_{n} \rightarrow \mathcal{F}_{P_{k}}$ defined by $\theta(x)=a e_{k n} x$ is a

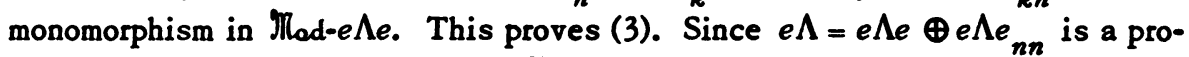
jective left $e \Lambda e$-module and since $\mathcal{F}_{j}, 1 \leq i \leq n$, is isomorphic to a right ideal of $e \Lambda e$, therefore the sequence $0 \rightarrow \mathcal{F J}_{i} \otimes_{e \Lambda e} e \Lambda \rightarrow e \Lambda e \otimes_{e \Lambda e} e \Lambda$ is exact. This yields $\mathcal{G F}_{J_{i}} \simeq\left(\mathcal{F}_{J_{i}}\right) e \Lambda$, so that $\mathrm{hd}_{\Lambda}\left(\mathcal{F}_{J_{i}}\right) e \Lambda=\mathrm{hd}_{\Lambda} \mathcal{G F}_{J_{i}}=\mathrm{hd}_{e \Lambda e} \mathcal{F}_{J_{i}}$, for $1 \leq i \leq n$, by Proposition 1.10(2). By using matrix multiplication one can easily check that $\left(\mathcal{F}_{i}\right) e \Lambda=J_{i}$ for $i \neq n, k$. This completes the proof of the lemma.

Proposition 2.3. Let $R$ be a local domain. Let $\Lambda=\left(\Lambda_{i j}\right) \subseteq M_{n}(R)$ be a

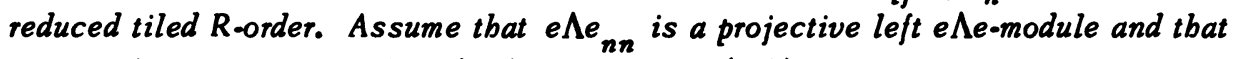
$g 1 \operatorname{dim} e \Lambda e<\infty$. Set $I=\Sigma_{i \neq n} \Lambda_{n i} \Lambda_{i n^{\circ}}$ If $g l \operatorname{dim}(R / I)<\infty$, then

(1) hd $J_{n} \leq$ hd $e \Delta e^{\mathcal{F}} J_{n}+g l \operatorname{dim}(R / I)$.

(2) $\mathrm{gl} \operatorname{dim} e \Lambda e \leq \mathrm{gl} \operatorname{dim} \Lambda \leq \mathrm{gl} \operatorname{dim} e \Lambda e+\mathrm{gl} \operatorname{dim}(R / I)+1$.

Proof. Since $\Lambda$ is reduced and $R$ is local, $I \subseteq \mathrm{m}$. Also, $J(\Lambda)$ is obtained from $\Lambda$ by replacing the diagonal entries $R$ by $\mathrm{m}$. Let $\mathrm{gl} \operatorname{dim} e \Lambda e=\alpha<\infty$ and $\mathrm{gl} \operatorname{dim}(R / l)=\beta<\infty$. By Lemma 2.1 we have a natural number $k \leq n$ and $0 \neq a$ in $R$ such that $\Lambda_{i n}=\Lambda_{i k} a$ for $1 \leq i \leq n-1$. Hence by Proposition 1.10(2), Lemma 2.2 and Lemma 0.1 we have

$$
\begin{aligned}
g 1 \operatorname{dim} e \Lambda e & \leq \mathrm{gl} \operatorname{dim} \Lambda, \\
\operatorname{hd}_{\Lambda} J_{i} & \leq a-1 \text { for } i \neq n, k, \\
\operatorname{hd}_{\Lambda}\left(\mathcal{F}_{J_{i}}\right) e \Lambda=\operatorname{hd}_{e \wedge e} \mathfrak{F}_{J_{i}} & \leq a-1 \text { for } i=n, k .
\end{aligned}
$$

Since $\Lambda_{k i} \Lambda_{i n}=\Lambda_{k i} \Lambda_{i k} a \subseteq$ ma for $i \neq k, n$, therefore by using matrix multiplications one shows that $\left(\mathcal{F}_{n}\right) e \Lambda$ is obtained from $J_{n}$ by replacing the $(n, n)$ th entry $m$ by $l$ and that $\left(F_{J_{k}}\right) e \Lambda$ is obtained from $J_{k}$ by replacing the $(k, n)$ th entry $R a$ by ma. Since $a \Lambda_{n i}=R a \Lambda_{n i}=\Lambda_{k n} \Lambda_{n i} \subseteq \Lambda_{k i}$ and $a \Lambda_{n k}=\Lambda_{k n} \Lambda_{n k} \subseteq \mathrm{m}$, therefore we have

$$
\left(\left(\mathcal{F}_{J_{k}}\right) e \Lambda\right)+a P_{n}=J_{k} \text { and }\left(\left(\mathcal{F}_{J_{k}}\right) e \Lambda\right) \cap a P_{n}=a J_{n} \cong J_{n}
$$

From this we get a short exact sequence

$$
0 \rightarrow a J_{n} \stackrel{\theta}{\rightarrow}\left(\left(\mathcal{F}_{J_{k}}\right) e \Lambda\right) \oplus a P_{n} \stackrel{\phi}{\rightarrow} J_{k} \rightarrow 0
$$

where $\theta(b)=(b, b)$ and $\phi\left(c, c^{\prime}\right)=c-c^{\prime}$. We now determine an upper bound on $\operatorname{hd}_{\Lambda} J_{n}$. As observed before $I \cong \mathrm{m}$. If $I=\mathfrak{m}$, then $\beta=0$ and $\left(\mathcal{F} J_{n}\right) e \Lambda=J_{n}$, so that $\mathrm{hd}_{\Lambda} J_{n}=\mathrm{hd}_{e \Lambda e} \mathcal{F}_{n} \leq \alpha-1$. Since $\mathrm{hd}_{\Lambda}\left(\mathcal{F}_{J_{k}}\right) e \Lambda \leq a-1$, therefore from the short exact sequence $(*)$ and Lemma 0.6 we have hd $J_{k} \leq \alpha$. Hence by Lemma 1.2 
we have $\mathrm{gl} \operatorname{dim} \Lambda \leq \alpha+1$. Now assume that $I \varsubsetneqq \mathrm{m}$. Since $\mathrm{gl} \operatorname{dim}(R / I)=\beta<\infty$ and $R$ is local, therefore $\bar{R}=R / l$ is a regular local ring of dimension $\beta \neq 0$ and having the unique maximal ideal $\bar{m}=\mathrm{m} / l$. Hence $\overline{\mathrm{m}}=\sum_{i=1}^{\beta} \bar{x}_{i} \bar{R}$ for some $\bar{R}$. sequence $\bar{x}_{1}, \ldots, \bar{x}_{\beta}$. This yields $\mathrm{m}=1+\sum_{i=1}^{\beta} x_{i} R$, and

$$
x_{\nu} R \cap\left(I+\sum_{i=1}^{\nu-1} x_{i} R\right)=x_{\nu}\left(I+\sum_{i=1}^{\nu-1} x_{i} R\right), \quad 1 \leq \nu \leq \beta .
$$

Set $E_{0}=\left(\mathcal{F}_{J_{n}}\right) e \Lambda$, and for $1 \leq \nu \leq \beta$ set

$$
E_{\nu}=x_{\nu} P_{n}+x_{\nu-1} P_{n}+\cdots+x_{1} P_{n}+E_{0}=x_{\nu} P_{n}+E_{\nu-1} \text {. }
$$

It follows that $E_{\beta}=J_{n}$. By using (\#) one gets that $E_{\nu} P_{n} \cap E_{\nu-1}=x_{\nu} E_{\nu-1}$ $E_{\nu-1}$, by Remarks 3 and 4 of $\$ 1$. Hence the two families $E_{\nu}, 0 \leq \nu \leq \beta$, and $x_{\nu} P_{n}, 1 \leq \nu \leq \beta$, satisfy the hypothesis of Lemma 1.1. Therefore hd $J_{n}=$ hd $_{\Lambda} E_{\beta}$ $\leq \operatorname{hd}_{\Lambda} E_{0}+\beta \leq \alpha-1+\beta$. Since $\operatorname{hd}_{\Lambda}\left(\mathcal{F}_{k}\right) e \Lambda \leq a-1$, therefore from the short exact sequence $(*)$ and Lemma 0.6 we get hd $J_{k} \leq \alpha+\beta$. Thus gl dim $\Lambda \leq \alpha+$ $\beta+1$, by Lemma 1.2. That $\mathrm{gl} \operatorname{dim} e \Lambda e \leq \mathrm{gl} \operatorname{dim} \Lambda$ follows from Proposition 1.10(2). This completes the proof of the proposition.

Corollary 2.4. Let $R$ be a local domain. Let $\Lambda=\left(\Lambda_{i j}\right) \subseteq M_{n}(R)$ be a reduced tiled $R$-order. Assume that $e \Lambda e_{n n}$ and $e_{n n} \Lambda e$ are projective left and right $e \Lambda e$ modules. If $\mathrm{gl} \operatorname{dim} e \Lambda e=\alpha<\infty$ and $\mathrm{gl} \operatorname{dim}(R / I)=\beta<\infty$, where $I=\Sigma_{i \neq n} \Lambda_{n i} \Lambda_{i n}$, then

(1) hd $_{\Lambda} J_{n} \leq \beta$.

(2) $\alpha \leq \mathrm{gl} \operatorname{dim} \Lambda \leq \sup (\beta+1, \alpha-1)+1$.

Proof. As $\mathfrak{F}_{n}=J_{n} e=e_{n n} \Lambda e$, (1) follows from Proposition 2.3(1). To prove (2) we use notations of the proof of Proposition 2.3(1). By Lemma 2.2 we have $\operatorname{hd}_{\Lambda} J_{i} \leq a-1$ for $i \neq n, k$, and $\operatorname{hd}_{\Lambda}\left(\mathcal{F}_{J_{k}}\right) e \Lambda \leq a-1$. If $\operatorname{hd}_{\mathbf{A}}\left(\mathcal{F}_{J_{k}}\right) e \Lambda \geq \operatorname{hd}_{\Lambda} J_{n}$ then, by using the short exact sequence $(*)$ of Proposition 2.3 and Lemma 0.6 , one gets that $\operatorname{hd}_{\Lambda} J_{k}=\operatorname{hd}_{\Lambda}\left(\mathcal{F}_{k}\right) e \Lambda \leq a-1$; and if hd ${ }_{\Lambda}\left(\mathcal{F}_{J_{k}}\right) e \Lambda \leq \operatorname{hd}_{\Lambda} J_{n}$, then $\operatorname{hd}_{\Lambda} J_{k} \leq 1+$ $\operatorname{hd}_{\Lambda} J_{n} \leq 1+\beta$. Thus hd $J(\Lambda) \leq \sup (\beta+1, \alpha-1)$. Now Lemma 1.2 and Proposition $1.10(2)$ complete the proof of the corollary.

In the next theorem we give a sufficient condition for a tiled $R$-order $\Lambda=$ $\left(\Lambda_{i j}\right) \subseteq M_{n}(R), R$ a local domain, to be of finite global dimension, and in Theorem 2.7 we remove the hypothesis that $R$ is local.

Theorem 2.5. Let $R$ be a commutative noetherian local domain with maximal ideal m. Let $\Lambda=\left(\Lambda_{i j}\right) \subseteq M_{n}(R)$ be a tiled $R$-order. Let $e=\Sigma_{i=1}^{n-1} e_{i i}$. If e $\Lambda_{n n}$ is a projective left e $\mathrm{e}$-module, then the following conditions are equivalent:

(1) $\mathrm{gl} \operatorname{dim} \Lambda<\infty$,

(2) $\mathrm{gl} \operatorname{dim} e \Lambda e<\infty$ and if $I=\Sigma_{i \neq n} \Lambda_{n i} \Lambda_{\text {in }}$ then $I=R$ or $\mathrm{gl} \operatorname{dim}(R / I)<\infty$. 
Furthermore, if these conditions bold, then $\mathrm{gl} \operatorname{dim} e \Lambda e \leq \mathrm{gl} \operatorname{dim} \Lambda \leq \mathrm{gl} \operatorname{dim} e \Lambda e$ $+g l \operatorname{dim}(R / I)+1$, where if $I=R$, then for the purpose of this theorem we set gl $\operatorname{dim}(R / I)=-1$.

Proof. By Lemma 2.1 we have a natural number $k \lesseqgtr n$ and $0 \neq a$ in $R$ such that $\Lambda_{i n}=\Lambda_{i k} a$ for $1 \leq i \leq n-1$. Hence $I=\Sigma_{i \neq n} \Lambda_{n i} \Lambda_{i n}=\Lambda_{n k} a$. Since $R$ is a local ring, $I \stackrel{i k}{=} R$ if and only if $\Lambda_{n j}=R=\Lambda_{j n}$ for some $J \neq n$. Hence, if $\Lambda$ is reduced, then $I \varsubsetneqq R$. We now prove $(1) \Rightarrow(2)$. By Proposition $1.10(2)$ we have gl $\operatorname{dim} e \Lambda e \leq \mathrm{gl} \operatorname{dim} \Lambda<\infty$. If $I=R$, then we are done. So assume that $\Lambda_{n k} a=$ $I \varsubsetneqq R$. Let $a$ be obtained from $\Lambda$ by replacing the $(n, n)$ th entry $R$ by $I$. One can easily check that $a$ is a two-sided ideal of $\Lambda$ and that $a^{2}=a$. Since $a=\bigoplus_{i=1}^{n-1} \Lambda e_{i i} \oplus \Lambda e_{k k} a$, $a$ is a projectvie left $\Lambda$-module. Hence, by Lemma 0.3, gl $\operatorname{dim} \Lambda / a<\infty$. Obviously, the map $\theta: \Lambda / a \rightarrow R / I$ defined by $\theta\left[\left(\lambda_{i j}\right)+a\right]=\lambda_{n n}$ $+I$ is a ring isomorphism. Hence $\mathrm{gl} \operatorname{dim}(R / l)<\infty$.

We now prove $(2) \Rightarrow(1)$ by using induction on $n$. Let $n=2$. Then $e \Lambda e \simeq R$, so that $\mathrm{gl} \operatorname{dim} R=\mathrm{gl} \operatorname{dim} e \Lambda e<\infty$. If $\Lambda$ is not reduced, then $\Lambda=M_{2}(R)$, and $\Lambda$ and $R$ are Morita equivalent. This yields $g l \operatorname{dim} R=g l \operatorname{dim} \Lambda<\infty$. If $\Lambda$ is reduced, then the theorem follows from Proposition 2.3. Let $n \geq 3$. Again if $\Lambda$ is reduced, then the theorem follows from Proposition 2.3. So assume that $\Lambda$ is not reduced. In that case we have natural numbers $s \underset{+}{l} \leq n$ such that $\Lambda_{s l}=R=\Lambda_{l s}$. But then $\Lambda_{s i} \supseteq \Lambda_{s l} \Lambda_{l i}=\Lambda_{l i} \supseteq \Lambda_{l s} \Lambda_{s i}=\Lambda_{s i}$, so that $\Lambda_{s i}=\Lambda_{l i}$ for $1 \leq i \leq n$. Similarly $\Lambda_{i s}=\Lambda_{i l}$ for $1 \leq i \leq n$. If $l=n$, then $\Lambda$ and $e \Lambda e$ are Morita equivalent and therefore $\mathrm{gl} \operatorname{dim} \Lambda=\mathrm{gl} \operatorname{dim} e \Lambda e<\infty$. If $l \Varangle n$, then consider $\Gamma$, the tiled $R$-order obtained from $\Lambda$ by deleting the $l$ th row and the $l$ th column. Clearly, $\Gamma \subseteq M_{n-1}(R)$, and $\Lambda$ and $\Gamma$ are Morita equivalent. Let $f=\Sigma_{i \neq n ; i \neq l} e_{i i}$. Then $e \Lambda e$ and $\tilde{\Gamma} f$ are Morita equivalent. Further $\int \Gamma e_{n n}$ is a projective left $f \Gamma f$-module, by Lemma 2.1 applied to $\Gamma$. Also, $I=\Sigma_{i \neq n ; i \neq l} \Lambda_{n i} \Lambda_{i n}$. Hence by the induction hypothesis we have $\mathrm{gl} \operatorname{dim} \Gamma<\infty$, and $\mathrm{gl} \operatorname{dim} \Gamma f \leq \mathrm{gl} \operatorname{dim} \Gamma \leq \mathrm{gl} \operatorname{dim} \Gamma f+$ $\mathrm{gl} \operatorname{dim}(R / I)+1$. Since $\mathrm{gl} \operatorname{dim} \Gamma f=\mathrm{gl} \operatorname{dim} e \Lambda e$ and $\mathrm{gl} \operatorname{dim} \Gamma=\mathrm{gl} \operatorname{dim} \Lambda$, the induction is complete. This completes the proof of the theorem.

Lemma 2.6. Let $R$ be a commutative noetberian domain. Let $I$ be a nonzero proper ideal of $R$. Assume that $\mathrm{gl} \operatorname{dim}\left(R_{\mathrm{m}} / I_{\mathrm{m}}\right)<\infty$ for all maximal ideals $\mathrm{m}$ of $R$ containing 1 . Then we bave:

(1) If $\mathrm{gl} \operatorname{dim} R=d<\infty$, then $\mathrm{gl} \operatorname{dim}(R / l) \leq d-1$.

(2) If $I$ is contained in only finitely many maximal ideals of $R$, then gl $\operatorname{dim}(R / I)<\infty$.

Proof. Let $\mathcal{S}$ be the set of all maximal ideals $m$ of $R$ containing $l$. Let $\bar{R}=R / I$. By Lemma 0.2 we have $g l \operatorname{dim} R_{\mathrm{m}} \leq \mathrm{gl} \operatorname{dim} R$ for all $\mathrm{m}$ in $\mathcal{S}$ and gl $\operatorname{dim} \bar{R}=\sup _{\mathfrak{m} \in \delta} g l \operatorname{dim} \bar{R}_{\bar{m}}$, where $\bar{m}=m / I$. It is easy to see that if $m$ is in $\delta$, 
then $I_{\mathrm{m}}$ is a nonzero proper ideal of $R_{\mathrm{m}}$ and $\bar{R}_{\overline{\mathrm{m}}} \cong R_{\mathrm{m}} / I_{\mathrm{m}}$. Thus (2) is obvious and (1) follows from 0.10 applied to $R_{m}$.

Theorem 2.7. Let $R$ be a commutative noetherian domain. Let $\Lambda=\left(\Lambda_{i j}\right) \subseteq$ $M_{n}(R)$ be a tiled $R$-order. Assume that $e \Lambda e_{n n}$ is a projective left e $\Lambda$ e-module. Let $I=\Sigma_{i \neq n} \Lambda_{n i} \Lambda_{i n}$. If $\mathrm{gl} \operatorname{dim} R<\infty$ or if $I$ is contained in only finitely many maximal ideals of $R$, then the following conditions are equivalent:

(1) $g l \operatorname{dim} \Lambda<\infty$,

(2) $\mathrm{gl} \operatorname{dim} e \Lambda e<\infty, I=R$ or $\mathrm{gl} \operatorname{dim}(R / I)<\infty$.

If these conditions bold then we have

$\mathrm{gl} \operatorname{dim} e \Lambda e \leq \mathrm{gl} \operatorname{dim} . \Lambda \leq \mathrm{gl} \operatorname{dim} e \Lambda e+g l \operatorname{dim}\left(R,{ }^{\prime} l\right)+1$,

where, if $I=R$, then for the purpose of this theorem we set $\mathrm{gl} \operatorname{dim}(R / I)=-1$.

Proof. The proof follows easily from the familiar localization, Lemmas 0.2 , 2.6 and Theorem 2.5.

We conclude this section with the following proposition.

Proposition 2.8. Let $\Lambda=\left(\Lambda_{i j}\right) \subseteq M_{n}(R)$ be a triangular tiled $R$-order over a commutative noetherian domain $R$. If $\mathrm{gl} \operatorname{dim} \Lambda<\infty$, then $\mathrm{gl} \operatorname{dim} R<\infty$.

Proof. Since $\Lambda$ is a triangular tiled $R$-order, $\Lambda_{i j}=R$ for $i \leq j$. Therefore $e \Lambda e_{n n}$ is a projective left $e \Lambda e$-module and $e \Lambda e \subseteq M_{n-1}(R)$ is a triangular tiled $R$-order. Hence by Proposition 1.10(2) we have $g l \operatorname{dim} e \Lambda e<\infty$. Applying this argument successively we conclude that $\mathrm{gl} \operatorname{dim} R<\infty$. This completes the proof.

In the next section we characterize triangular tiled $R$-orders of finite global dimension, where $R$ is a commutative noetherian domain of finite global dimension.

3. Main theorem. In this section we investigate the global dimension of a tiled $R$-order $\Lambda \subseteq M_{n}(R)$ over a commutative noetherian domain $R$ of finite global dimension. In view of Lemma 0.2 , the main case we have to deal with is when $R$ is a regular local ring. In the first half of this section we derive some of the properties of a tiled $R$-order over a regular local ring $R$. We recall that when $R$ is a local ring, then the maximal ideal is denoted by $m$.

Lemma 3.1. Let $R$ be a regular local ring of dimension $d$ and let $\Lambda=\left(\Lambda_{i j}\right)$ $\subseteq M_{n}(R)$ be a tiled R-order. Then, gl $\operatorname{dim} \Lambda \geq d$.

Proof. Let $A=\Sigma_{i=1}^{n} R u_{i}$ be a free left $R$-module on the basis $u_{1}, u_{2}, \cdots, u_{n}$. Treat $A$ as a right $\Lambda$-module naturally. Let $h_{\Lambda} A=\alpha$. If $a=\infty$, then we are done. So assume that $a<\infty$. Let $m$ be generated by an $R$-sequence $x_{1}, x_{2}, \cdots$, $x_{d}$, i.e., $\mathrm{m}=\sum_{i=1}^{d} x_{i} R$. Let $X_{i}=\operatorname{diag} x_{i}, 1 \leq i \leq d$. Then the matrices $x_{i}$ are nonzero divisors in $\Lambda$ and are contained in $J(\Lambda)$, by Lemma 1.3. Furthermore, 
$X_{i}$ is a nonzero divisor on the right $\Lambda$-module $A / \Sigma_{j=1}^{i-1} A X_{j}$. Since hd $A=\alpha<\infty$, $\operatorname{hd}_{\Lambda}\left(A / A X_{1}\right)=a+1$, by Lemmas 0.7 and 0.8 . An easy induction shows that $\operatorname{hd}_{\Lambda}\left(A / \Sigma_{j=1}^{d} A X_{j}\right)=a+d$. This completes the proof of the lemma.

Remark. The techniques of the proof of the above lemma are similar to those used in the proof of Theorem 14 of [17]. The above lemma also follows from Corollary 3.2 of [20], but our proof is elementary. The notations established in Lemma 3.1 will be needed in the proof of Theorem 3.4 of this paper.

Lemma 3.2. Let $\Lambda=\left(\Lambda_{i j}\right) \subseteq M_{n}(R)$ be a reduced triangular tiled $R$-order, where $R$ is a regular local ring of dimension $d$. Let $\mathrm{m}$ be generated by an $R$. sequence $x_{1}, x_{2}, \cdots, x_{d}$. Assume that $\Lambda_{i j}=x_{1} R$ whenever $i=n$ or $n-1$ and $i \geqslant j$. Then hd $J_{i} \leq d-1$ for $i=n-1$, n. Hence, if $n=2$, then $\mathrm{gl} \operatorname{dim} \Lambda=d$, and if $d=2$, then hd $J_{i}=1$ for $i=n-1, n$.

Proof. Since $\Lambda$ is reduced, $J(\Lambda)$ is obtained from $\Lambda$ by replacing the diagonal entries $R$ by $m$. Set $E_{1}=P_{n}$ and $E_{\nu}=x_{\nu} P_{n-1}+x_{\nu-1} P_{n-1}+\cdots+x_{2} P_{n-1}+P_{n}$ for $2 \leq \nu \leq d$. Then $E_{d}=J_{n-1}$ and $E_{\nu}=x_{\nu} P_{n-1}+E_{\nu-1}$. Since $x_{1}, \ldots, x_{d}$ is an $R$-sequence, therefore $x_{\nu} R \cap \Sigma_{i=1}^{\nu-1} x_{i} R=x_{\nu} \sum_{i}^{\nu=1} x_{i} R$ for $2 \leq \nu \leq d$. It follows that $x_{\nu} P_{n-1} \cap E_{\nu-1}=x_{\nu} E_{\nu-1} \simeq E_{\nu-1}$ for $2 \leq \nu \leq d$. Since hd $E_{1}=0$, therefore Lemma 1.1, applied to the two families $E_{\nu}, 1 \leq \nu \leq d$, and $x_{\nu} P_{n-1}$, $2 \leq \nu \leq d$, of right $\Lambda$-modules, yields $\mathrm{hd}_{\Lambda} J_{n-1}=\mathrm{hd}_{\Lambda} E_{d} \leq d-1$. Similarly to show that hd $J_{n} \leq d-1$, set $F_{1}=x_{1} P_{1}$ and $F_{\nu}=x_{\nu} P_{n}+\cdots+x_{2} P_{n}+x_{1} P_{1}$ for $2 \leq \nu \leq d$. Then $F_{\nu}=x_{\nu} P_{n}+F_{\nu-1}$ and $F_{d}=J_{n}$. An argument similar to the above shows that hd $J_{n} \leq d-1$. It is easy to see that when $d=2, J_{n-1}$ and $J_{n}$ are not projective right $\Lambda$-modules. Therefore we must have hd $J_{i}=1$ for $i=n-1$ and $n$. The remaining assertion follows from Lemmas 1.2 and 3.1. This completes the proof.

Proposition 3.3. Let $\Lambda=\left(\Lambda_{i j}\right) \subseteq M_{n}(R)$ be a tiled $R$-order, where $R$ is a regular local ring of dimension $d$, with the unique maximal ideal $m$ generated by an R-sequence $x_{1}, x_{2}, \ldots, x_{d}$. Assume that $\Lambda_{1 j}=R$ for all $j$ and $\Lambda_{i j}=x_{1} R$ or $R$ whenever $i \neq 1$ or $i \neq j$. Then:

(1) $\mathrm{gl} \operatorname{dim} \Lambda \leq d(n-1)$.

(2) If $\Lambda_{i j}=x_{1} R$ whenever $i \neq j$ and $i \neq 1$, then $\operatorname{gl} \operatorname{dim} \Lambda \leq d+1$.

(3) If $\Lambda$ is a triangular tiled $R$-order then $\mathrm{gl} \operatorname{dim} \Lambda=d$.

Proof. First consider the case $n=2$. If $\Lambda$ is reduced then $g 1 \operatorname{dim} \Lambda=d$, by Lemma 3.2. If $\Lambda$ is not reduced then $\Lambda=M_{2}(R)$, so that $\mathrm{gl} \operatorname{dim} \Lambda=d=\mathrm{gl} \operatorname{dim} R$. Thus, all the assertions are true for $n=2$.

We now prove (3) by using induction on $n$. Let $n \geq 3$. If $\Lambda$ is not reduced then $\Lambda$ is Morita equivalent to a triangular tiled $R$-order in $M_{n-1}(R)$ satisfying 
the hypothesis of the proposition. Hence by the induction hypothesis we have gl $\operatorname{dim} \Lambda=d$. Now assume that $\Lambda$ is reduced. Since $\Lambda$ is a triangular tiled $R$-order $e \Lambda e_{n n} \cong e \Lambda e_{n-1, n-1}$ as left $e \Lambda e$-modules. Also, $e \Lambda e \subseteq M_{n-1}(R)$ satisfies the hypothesis of the proposition. Hence by Lemmas 1.2, 2.2 and the induction hypothesis we have hd $J_{i} \leq d-1$ for $i \leq n-2$. Also, by Lemma 3.2 we have $\operatorname{hd}_{\Lambda} J_{i} \leq d-1$ for $i=n-1, n$. Thus by Lemmas 1.2 and 3.1 we have $g 1 \operatorname{dim} \Lambda=d$. This completes the induction.

We now prove (1) and (2) simultaneously by using induction on $n$. Let $n \geq 3$. First assume that $\Lambda$ is reduced. Since $\Lambda_{1 j}=R$ for all $j$, therefore by Lemma 1.7 we have a natural number $l \geqslant 1$ such that $\Lambda_{l i} \neq R$ whenever $i \neq l$, so that $\Lambda_{l i}=x_{1} R$ for $i \neq l$. Since the global dimension of a ring is isomorphism invariant, we may assume that $l=2$. Let $y=\left(y_{i j}\right)$ be in $M_{n}(K)$, where $y_{12}=x_{1}^{-1}, y_{21}=1$ $=y_{i i}$ for $i \geq 3, y_{i j}=0$ otherwise. Let $\Gamma=\left(\Gamma_{i j}\right)=y \Lambda y^{-1}$. Computation shows that $\Gamma_{1 j}=R$ for all $j ; \Gamma_{2 j}=R$ for $j \geq 2 ; \Gamma_{i 1}=x_{1} \Lambda_{i 2}$ for $i \geq 2 ; \Gamma_{i 2}=x_{1} R$ for $i \geq 3 ; \Gamma_{i j}=\Lambda_{i j}$ otherwise. Hence $\Gamma=\left(\Gamma_{i j}\right) \subseteq M_{n}(R)$ is a tiled $R$-order and $e_{11} \Gamma e^{\prime}$ is a projective right $e^{\prime} \Gamma e^{\prime}$-module, where $e^{\prime}=\Sigma_{i=2}^{n} e_{i i}$. Further, $I^{\prime}=\Sigma_{i \neq 1} \Gamma_{1 i} \Gamma_{i 1}=x_{1} R$, so that $g l \operatorname{dim}\left(R / I^{\prime}\right)=d-1<\infty$. Clearly $e^{\prime} \Gamma e^{\prime} \subseteq$ $M_{n-1}(R)$ satisfies the hypothesis of the proposition in cases (1) and (2). We now complete the proof of (1). By the induction hypothesis $\mathrm{gl} \operatorname{dim} e^{\prime} \Gamma e^{\prime} \leq d(n-2)$; hence by the analogue of Theorem 2.5 we have $\mathrm{gl} \operatorname{dim} \Gamma \leq d(n-2)+d=d(n-1)$. Since $\Lambda$ and $\Gamma$ are conjugate, gl $\operatorname{dim} \Lambda \leq d(n-1)$. For (2) we observe that by the hypothesis we have $\Lambda_{i 2}=x_{1} R$ for all $i \geq 3$. Thus $\Gamma_{i 1}=\Gamma_{i 2} x_{1}$ for $i \geq 2$. Hence by Lemma 2.1 (rather, its analogue), we have that $e^{\prime} \Gamma e_{11}$ is a projective left $e^{\prime} \Gamma e^{\prime}$-module. Therefore by the analogue of Corollary 2.4 and the induction hypothesis we have

$$
\text { gl dim } \Gamma \leq \sup (d-1+1, d+1-1)+1=d+1 .
$$

Thus $\mathrm{gl} \operatorname{dim} \Lambda=g l \operatorname{dim} \Gamma \leq d+1$.

For the case $\Lambda$ is not reduced, the argument is similar to the case (3) and we leave it to the reader. This completes the induction and the proof of the proposition too.

The author is thankful to the referee for suggesting the following corollary.

Corollary 3.4. Let $R$ be a regular local ring of dimension $d, \Lambda$ a triangular tiled R-order satisfying the bypothesis of Proposition 3.3. Then each finitely generated $R$-free $\Lambda$-module is projective.

Proof. Follows from Proposition 3.3 of this paper and Proposition 3.5 of [20].

Theorem 3.5. Let $R$ be a commutative noetherian domain of global dimension $d<\infty$. Let I be a nonzero proper ideal of $R$ such that $\mathrm{gl} \operatorname{dim}(R / I)<\infty$. Let 
$\Lambda=\left(\Lambda_{i j}\right) \subseteq M_{n}(R)$ be a tiled $R$-order. Assume that $\Lambda_{1 j}=R$ for all $j$ and $\Lambda_{i j}=I$ or $R$ whenever $i \neq j$ and $i \neq 1$. Then:

(1) gl $\operatorname{dim} \Lambda \leq d(n-1)$.

(2) If $\Lambda$ is a triangular tiled $R$-order, then $\mathrm{gl} \operatorname{dim} \Lambda=d$.

(3) If $\Lambda_{i j}=I$ whenever $i \neq j$ and $i \neq 1$, then $\mathrm{gl} \operatorname{dim} \Lambda \leq d+1$. Furthermore, if $R$ is local, $I=J(R)$ and $n \geq 3$, then $\mathrm{gl} \operatorname{dim} \Lambda=d+1$.

(4) If $n \geq 4, R$ is local and if $\Lambda_{i j}=I$ whenever $i \neq j$ and $i \neq 1$, then $\Lambda$ is not conjugate to a triangular tiled $R$ - order.

Proof. Since $\mathrm{gl} \operatorname{dim} R=d, \mathrm{gl} \operatorname{dim} R_{\mathrm{m}} \leq d$ for all maximal ideals $\mathrm{m}$ of $R$ with equality occurring for at least one $m$. By Lemma 0.2 we have gl dim $\Lambda=$ $\sup _{m} g l \operatorname{dim} \Lambda_{m}$, where the supremum is taken over all maximal ideals $m$ of $R$. If $I \notin \mathbb{m}$, then $I_{\mathrm{m}}=R_{\mathrm{m}}$, and therefore $\Lambda_{\mathrm{m}}=M_{n}\left(R_{\mathrm{m}}\right)$. Thus if $I \nsubseteq \mathbb{m}$, then gl $\operatorname{dim} \Lambda_{\mathrm{m}}=g l \operatorname{dim} R_{\mathrm{m}}$. If $I \subseteq \mathrm{m}$, then $\mathrm{gl} \operatorname{dim}\left(R_{\mathrm{m}} / I_{\mathrm{m}}\right) \leq \mathrm{gl} \operatorname{dim}(R / I)<\infty$. Thus to prove the theorem we may assume that $R$ is a regular local ring of dimension $d$ with maximal ideal $m$. But then, by Lemma 0.9 , we have an $R$-sequence $x_{1}, x_{2}, \ldots, x_{d}$ such that $I=\Sigma_{i=1}^{\mu} x_{i} R, \mu \geq 1$, and $m=\sum_{i=1}^{d} x_{i} R$. Also, we have that $R /\left(x_{\mu}\right)$ is a regular local ring of dimension $d-1$ and $x_{i}+\left(x_{\mu}\right), 1 \leq i \leq d$, $i \neq \mu$, is an $R /\left(x_{\mu}\right)$-sequence generating the maximal ideal of $R /\left(x_{\mu}\right)$. Set $a=\left(a_{i j}\right) \subseteq \Lambda$, where $a_{i j}=\left(x_{\mu}\right)$ for $1 \leq i, j \leq n$. Clearly $a$ is a two-sided ideal contained in $J(\Lambda)$ and is projective as a right $\Lambda$-module. Hence, by Lemma 0.4 , we have

$$
\text { gl } \operatorname{dim} \Lambda \leq 1+g l \operatorname{dim} \Lambda / a .
$$

It is easy to see that $\Lambda / a$ is isomorphic to a tiled $R /\left(x_{\mu}\right)$-order $\left(\Lambda_{i j} /\left(x_{\mu}\right)\right) \subseteq$ $M_{n}\left(R /\left(x_{\mu}\right)\right)$. We now prove (1), (2) and the first part of (3) simultaneously by using induction on $\mu$. For $\mu=1$ the assertions follow from Proposition 3.3. Let $\mu \geq 2$. By the induction hypothesis we have

$$
\begin{aligned}
\mathrm{gl} \operatorname{dim}(\Lambda / a) & \leq(d-1)(n-1) & & \text { in case (1), } \\
& =d-1 & & \text { in case (2), } \\
& \leq d & & \text { in case (3). }
\end{aligned}
$$

The assertions now follow from (\#\#) and Lemma 3.1.

We now prove the remaining part of (3). Since $\Lambda$ is reduced, $J(\Lambda)$ is obtained from $\Lambda$ by replacing the diagonal entries $R$ by $m$. Hence, for $i \geq 2, J_{i} \simeq$ $\Sigma_{i=1}^{d} P_{1} X_{i}$ as right $\Lambda$-modules, where $X_{i}=\operatorname{diag} x_{i}$. Since $P_{1}$ is projective and is isomorphic to $A$, where $A$ is a defined in Lemma 3.1, we have $\operatorname{hd}_{\Lambda}\left(P_{1} / \sum_{i=1}^{d} P_{1} X_{i}\right)=d$. Hence hd $J_{i}=d-1$ whenever $i \geq 2$. To complete the proof we show that hd $J_{1}=d$, since then by Lemma 1.2 we have gl $\operatorname{dim} \Lambda=d+1$. Set $E_{\nu}=P_{2}+P_{3}+\cdots+P_{\nu}$ for $2 \leq \nu \leq n$. Then, since $n \geq 3, E_{n}=J_{1}$; also 
for $3 \leq \nu \leq n$ we have $E_{\nu}=E_{\nu-1}+P_{\nu}, E_{\nu-1} \cap P_{\nu} \simeq J_{2}$ and $E_{\nu}$ is not projective as a right $\Lambda$-module. Hence, using short exact sequences, induction and Lemma 0.6 , one gets that $\mathrm{hd}_{\Lambda} E_{\nu}=d$ for $\nu \geq 3$. Thus hd $J_{1}=d$.

Lastly we prove (4). Let $n \geq 4$. Let $\bar{\Lambda}, \bar{P}_{i}=\bar{e}_{i i} \bar{\Lambda}, 1 \leq i \leq n$, be as in Proposition 1.9. Since $\Lambda$ is reduced, by Lemma $1.3, J(\bar{\Lambda})$ is obtained from $\bar{\Lambda}$ by replacing the diagonal entries $R / \mathrm{m}$ by zero. Computation shows that $\bar{P}_{i} J^{3}(\bar{\Lambda})=0$ for $1 \leq i \leq n$. Since $n \geq 4$, therefore none of $\bar{P}_{j}$ satisfies the condition $(*)$ of Proposition 1.9. Thus, by the same proposition, $\Lambda$ is not conjugate to a triangular tiled $R$-order.

Remark 1. The assertion (2) in the above theorem is a generalization of Theorem 14 of [17].

Remark 2. The assertion (4) in the above theorem is not true in general when $n \lesseqgtr 4$. For if $n=2$, then $\Lambda$ is itself a triangular tiled $R$-order; and if $n=3$ and $I=x R$ is a principal ideal, then $y \Lambda y^{-1}$ is a triangular tiled $R$-order, where $y=$ $\left(y_{i j}\right)$ is in $M_{3}(K)$, with $y_{12}=x^{-1}, y_{21}=y_{33}=1$ and $y_{i j}=0$ otherwise.

The next theorem characterizes triangular tiled $R$-orders of finite global dimension over a commutative noetherian domain $R$ of finite global dimension.

Theorem 3.6. Let $R$ be a commutative noetberian domain of global dimension $d<\infty$. Then the following three conditions on a triangular tiled $R$-order $\Lambda=\left(\Lambda_{i j}\right)$ $\subseteq M_{n}(R)$ are equivalent:

(1) $\mathrm{gl} \operatorname{dim} \Lambda<\infty$.

(2) $\Lambda_{i, i-1}=R$ or $g 1 \operatorname{dim}\left(R / \Lambda_{i, i-1}\right)<\infty$ for $2 \leq i \leq n$.

(3) gl $\operatorname{dim} \Lambda \leq d(n-1)$.

Proof. First we note that $e \Lambda e_{n n}$ is a projective left $e \Lambda e$-module, and since $\Lambda_{n i}=\Lambda_{n i} \Lambda_{i, n-1} \subseteq \Lambda_{n, n-1}$ for $i \neq n$, we have $I=\Sigma_{i \neq n} \Lambda_{n i} \Lambda_{i n}=\Lambda_{n, n-1}$. We now prove $(1) \Rightarrow(2)$ by using an induction on $n$. If $n=2$, then the assertion follows from Theorem 2.7. Let $n \geq 3$. Again by the same theorem we have gl dim $e \Lambda e<\infty$, and $\Lambda_{n, n-1}=R$ or $\mathrm{gl} \operatorname{dim}\left(R / \Lambda_{n, n-1}\right)<\infty$. Now the induction hypothesis completes the proof as $e \Lambda e$ is a triangular tiled $R$-order contained in $M_{n-1}(R)$. We now prove $(2) \Rightarrow(3)$ again by using an induction on $n$. Let $n=2$. Then, by Theorem 3.5(2), we have $g l \operatorname{dim} \Lambda=d$. Let $n \geq 3$. By the induction hypothesis we have gl dim $e \Lambda e \leq d(n-2)$. Hence by Theorem 2.7 and Lemma 2.6(1) we have gl $\operatorname{dim} \Lambda \leq \mathrm{gl} \operatorname{dim} e \Lambda e+g 1 \operatorname{dim}\left(R / \Lambda_{n, n-1}\right)+1 \leq d(n-2)+d-1+1=d(n-1)$.

(3) $\rightarrow(1)$ is trivial.

This completes the proof of the theorem.

Remark. The above theorem is a generalization of Theorem 1 of [8], [9]. 
Corollary 3.7. Let $R$ be a Dedekind domain. Let $\Lambda=\left(\Lambda_{i j}\right) \subseteq M_{n}(R)$ be a triangular tiled $R$-order. The following conditions are equivalent:

(1) gl $\operatorname{dim} \Lambda<\infty$.

(2) $\Lambda_{i, i-1}=R$ or $\Lambda_{i, i-1}$ is a product of distinct maximal ideals of $R$.

(3) $g l \operatorname{dim} \Lambda \leq n-1$.

It is known that the upper bound $d(n-1)$ in Theorem 3.6 is attained when $d=1([9$, Theorem 2], [17, Theorem 11]). By constructing an example we show that this is also the case when $d=2$.

Proposition 3.8. Let $R$ be a regular local ring of dimension 2. Let $\mathfrak{m}$ be the unique maximal ideal of $R$ generated by an $R$-sequence $x, y$. Let $\Lambda=\left(\Lambda_{i j}\right) \subseteq$ $M_{n}(R), n \geq 2$, where $\Lambda_{i j}=R$ whenever $i \leq j, \Lambda_{i, i-1}=x R$ for $2 \leq i \leq n$, and $\Lambda_{i j}=x \mathfrak{m}=x^{2} R+x y R$ otherwise. Then $\Lambda$ is a triangular tiled $R$-order in $M_{n}(R)$ and

$$
\begin{aligned}
& \operatorname{hd}_{\Lambda} J_{i}=2 i-1 \text { for } 1 \leq i \leq n-1, \\
& \operatorname{hd}_{\Lambda} J_{n}=\sup (2(n-2), 1) .
\end{aligned}
$$

Thus gl dim $\Lambda=2(n-1)$.

Proof. Computation shows that $\Lambda$ is a ring, hence is a triangular tiled $R$-order. Obviously $\Lambda$ is reduced; hence $J(\Lambda)$ is obtained from $\Lambda$ by replacing the diagonal entries $R$ by $m$. To prove the remaining assertions we use an induction on $n$. If $n=2$, then the assertions follow from Lemma 3.2. Let $n \geq 3$. Clearly $e \Lambda e$ satisfies the hypothesis of the proposition. Hence by the induction hypothesis we have $\mathrm{gl} \operatorname{dim} e \Lambda e=2(n-2)$. Since $e \Lambda e_{n n} \simeq e \Lambda e_{n-1, n-1}$, therefore by Proposition 1.10(1), Lemma 2.2 and the induction hypothesis we have hd $\mathrm{J}_{i}=$ hd $_{e A e} \mathcal{F}_{i}=2 i-1$ for $1 \leq i \leq n-2$, hd $_{\Lambda}\left(\mathcal{F}_{J_{n-1}}\right) e \Lambda=h_{e A e} \mathcal{F}_{n-1}=\sup (2(n-3), 1)$. Let $L_{1}=y P_{n-1}+P_{n}$ and $L_{2}=x P_{n-2}+P_{n}$. Then $L_{1}$ and $L_{2}$ are isomorphic to right ideals of $\Lambda$. Also one can easily check that $y P_{n-1} \cap P_{n}=y L_{2} \simeq L_{2}$ and $x P_{n-2} \cap P_{n}=x J_{n-2} \simeq J_{n-2}$. Thus by using obvious short exact sequences and Lemma 0.6 one concludes that hd ${ }_{\Lambda} L_{1}=$ hd $_{\Lambda} J_{n-2}+2=2(n-2)+1=2 n-3$. Hence, by Lemma 0.1 , we have gl $\operatorname{dim} \Lambda \geq 2 n-2=2(n-1)$. But then Theorem 3.6 yields $\mathrm{gl} \operatorname{dim} \Lambda=2(n-1)$. Since $I=\Sigma_{i \neq n} \Lambda_{n i} \Lambda_{i n}=x R, g l \operatorname{dim}(R / I)=1$. Therefore, by Lemmas $0.1,2.2$ and Proposition 2.3, we have hd $J_{n} \leq$ hd ${ }_{e \Delta e} \mathcal{F}_{n}+$ $1 \leq 2(n-2)=2(n-1) 2$. Hence, by Lemma 1.2 we must have hd $J_{n-1}=$ $2(n-1)-1$. To complete the induction we show that hd $J_{n}=2(n-1)-2$. Computation shows that $\left(\mathcal{F}_{n-1}\right) e \Lambda+P_{n}=J_{n-1}$ and $\left(\mathcal{F}_{n-1}\right) e \Lambda \cap P_{n}=J_{n}$. Hence the sequence

$$
0 \rightarrow J_{n} \stackrel{\theta}{\rightarrow}\left(\left(\mathcal{F}_{J_{n-1}}\right) e \Lambda\right) \oplus P_{n} \stackrel{\phi}{\rightarrow} J_{n-1}-0
$$

is exact, where $\theta(a)=(a, a)$ and $\phi(b, c)=b-c$. But $\operatorname{hd}_{\Lambda} J_{n-1}=2(n-1)-1$ 
and hd ${ }_{\Lambda}\left(F_{n-1}\right) e \Lambda=\sup (2(n-3), 1)$; therefore we must have hd $J_{n}=2(n-1)-2$, by Lemma 0.6 . This completes the induction and proves the proposition.

\section{BIBLIOGRAPHY}

1. M. Auslander and O. Goldman, Maximal orders, Trans. Amer. Math. Soc. 97 (1960), 1-24. MR 22 \#8034.

2. H. Bass, Algebraic K-theory, Benjamin, New York, 1968. MR 40 \#2736.

3. N. Bourbaki, Eléments de mathématique. XXIII. Part. 1. Les structures fondamentales de l'analyse. Livre II: Algébre. Chap. 8: Modules et anneaux semi-simples, Actualités Sci. Indust., no. 1261, Hermann, Paris, 1958. MR 20 \#4576.

4. K. L. Fields, $O_{n}$ the global dimension of residue rings, Pacific J. Math. 32 (1970), 345-349. MR $42 \# 6049$.

5. Manabu Harada, Note on the dimension of modules and algebras, J. Inst. Polytech. Osaka City Univ. Ser. A 7 (1956), 17-27. MR 18, 375.

6. N. Jacobson, Structure of rings, 2nd ed., Amer. Math. Soc. Colloq. Publ., vol. 37, Amer. Math. Soc., Providence, R. I., 1964. MR 36 \#5158.

7. A. V. Jategaonkar, Unpublished Lecture Notes, Cornell University, Ithaca, N. Y., 1971.

8. Vasanti A. Jategaonkar, Global dimension of triangular orders over DVR, Notices Amer. Math. Soc. 18 (1971), 626. Abstract \#71T-A106.

9. - Global dimension of triangular orders over a discrete v:luation ring, Proc. Amer. Math. Soc. 38 (1973), 8-14.

10. —- Tiled orders, Thesis, Cornell University, Ithaca, N. Y., 1973.

11. Irving Kaplansky, Fields and rings, Univ. of Chicago Press, Chicago, Ill., 1969. MR $42 \# 4345$.

12. J. Lambek, Lectures on rings and modules, Blaisdell, Waltham, Mass., 1966. MR 34 \#5857.

13. Bruno J. Mueller, On semiperfect rings, Illinois J. Math. 14 (1970), 464-467. MR 41 \#6909.

14. Joseph J. Rotman, Notes on homological algebra, Van Nostrand Reinhold, New York, 1968.

15. L. Silver, Noncommutative localizations and applications, J. Algebra 7 (1967), 44-76. MR $36 \# 205$.

16. L. W. Small, A change of rings theorem, Proc. Amer. Math. Soc. 19 (1968), 662666. MR $36 \# 6460$.

17. R. B. Tarsey, Global dimension of orders, Trans. Amer. Math. Soc. 151 (1970), 335-340.

18. - Global dimension of triangular orders, Proc. Amer. Math. Soc. 28 (1971), 423-426.

19. O. Zariski and P. Samuel, Commutative algebra. Vol. II, University Series in Higher Math., Van Nostrand, Princeton, N. J., 1960. MR 22 \#11006.

20. M. Ramaras, Maximal orders over regular local rings of dimension two, Trans. Amer. Math. Soc. 142 (1969), 457-479.

DEP ARTMENT OF MATHEMATICS, CORNELL UNIVERSITY, ITHACA, NEW YORK 14850 